\title{
Prevalence of Methicillin-Resistant Staphylococcus aureus Among Health Care Workers of Shri Satya Sai Medical College and Hospital -A Tertiary Care Centre.
}

\author{
Kalyani.K ${ }^{1}$, Karthika Jayakumar ${ }^{2}$, Sunil Kumar.J ${ }^{3}$ \\ ${ }_{1,2,3}$ (Department of Microbiology, Shri Sathya Sai Medical College and Research Institute,India.)
}

\begin{abstract}
Methicillin-resistant Staphylococcus aureus (MRSA) is an important cause of hospital-acquired infections in many countries. Mainly healthcare workers (HCWs) are at risk for Methicillin-resistant Staphylococcus aureus (MRSA) carriage. A total of 120 healthcare workers (HCWs) were screened for carriage of MRSA. Swabs of both anterior nares and web spaces of the hands and palms were taken, transported and incubated in Tween 80 at $35^{\circ} \mathrm{C}$ overnight aerobically before inoculation onto Mannitol Salt Agar (MSA). Inoculated MSA were incubated aerobically at $35^{\circ} \mathrm{C}$ for 18-24 hrs. Staphylococcus aureus was identified as Gram positive cocci with positive catalase, coagulase and DNAse test. MRSA were identified at different sites using oxacillin and cefoxitin discs diffusion methods. Overall carriage rate was $15.8 \%$., with the highest rate in nurses (7.5\%) while the lowest in Doctors (2.5\%). All the MRSA isolates were sensitive to all antibiotics. Vancomycin resistant strain was not identified. The commonest site of colonization was the anterior nares. Prolonged hospital stay and improper hand washing were found to be the major risk factors for MRSA colonization, although it would require screening of larger numbers before arriving at any definite conclusions. There is need for MRSA control policies in hospitals.
\end{abstract}

Keywords- Healthcare workers, Methicillin-resistant Staphylococcus aureus carriage, MRSA carriage, Nasal carriage, Staphylococcus aureus.

\section{Introduction}

Staphylococcal infections cause significant morbidity and mortality in both community and hospital settings. Treatment of infection caused by S.aureus has become more problematic since the development of antimicrobial resistant Staphylococcus aureus, Methicilin-Resistant Staphylococcus aureus (MRSA) ${ }^{[1] .}$.

MRSA has become endemic worldwide within the past two decades ${ }^{[2]}$. MRSA by definition is a strain of $S$. aureus that is resistant to a large group of antibiotics called "the beta-lactams", which include the Penicillins and the Cephalosporins. MRSA is often sub-categorized as Community-Associated MRSA (CAMRSA) or Health Care-Associated MRSA (HA-MRSA) ${ }^{[3] .}$ MRSA was discovered in 1961 in the United Kingdom. It made its first major appearance in the United States in 1981 among intravenous drug users. MRSA is often referred as a "Super bug". Methicillin resistance is not confined to S. aureus. During the 1980s, attention was drawn to the high incidence of "Methicillin-resistant Staphylococcus epidermidis (MRSE) in nosocomial infections. The MRSA is encoded by $\boldsymbol{m e c} \mathbf{A}$ gene . The main mode of MRSA transmission is from MRSA-colonized or infected patients to another by direct contact, through touch of health care workers ${ }^{[4]}$.Thus, patients may acquire MRSA from colonized HCWs ${ }^{[5]}$. Nasal carriage is a major risk factor for Staphylococcus aureus infection, especially for MRSA ${ }^{[3]}$. Anterior nares are the major reservoir of S. aureus: $20 \%$ of humans are persistently and asymptomatically colonized, $60 \%$ are intermittently, and $20 \%$ are noncarriers ${ }^{[6] .}$

The role of MRSA carriers in the transmission of this pathogen is critical. Therefore, healthcare workers who are at the interface between the hospital and the community may serve as agents of crosstransmission of HA-MRSA and CA-MRSA ${ }^{[2] .}$

Nosocomial transmissions of MRSA from health care workers to hospitalised patients have been documented. Similarly, screening and eradication of MRSA from colonized healthcare workers have been recognised and recommended as an important part of a comprehensive infection control policy for this organism [2]. It was with this background that this study was undertaken in our hospital mainly to screen health care workers for MRSA carriage, and to identify risk factors for MRSA colonization.

\section{Materials \& Methods}

2.1 Study duration and population: This study was conducted at SSSMCH, Kancheepuram District, and Tamil Nadu for 2 months period between May-July2011.

2.2 Clinical isolates: A total of 120 subjects ( 40 doctors, 65 nursing staff and 15 technicians) were screened for MRSA after obtaining informed consent and approval by the institute ethical committee. Complete information 
about the participants were obtained in a Proforma designed for this purpose, after getting their consent. Mainly HCWs of Hospital are Included in the study as they are more prone for colonization of MRSA.

2.3 Specimen collection: Swabs of both anterior nares of consenting persons were taken with a sterile swab stick moistened with sterile physiological saline and transported in Tween 80 to the Microbiology laboratory unit of the hospital for analysis. Similar swabs of the palms and web spaces of the hands were also taken after at least $30 \mathrm{~min}$ of the last hand wash.

The swabs in Tween 80 were incubated at $35^{\circ} \mathrm{C}$ overnight before being inoculated onto Mannitol Salt Agar (MSA). Inoculated MSA were incubated at $35^{\circ} \mathrm{C}$ for $18-24 \mathrm{~h}$. Gram positive cocci isolates which are catalase, coagulase \& DNAse positive using standard techniques, were identified as Staphylococcus aureus and were screened for methicillin resistance by modified Kirby-Bauer method using Oxacillin ( $1 \mu \mathrm{g})$ and Cefoxitin $(30 \mu \mathrm{g})$ disk diffusion methods on Mueller-Hinton Agar (MHA)

2.4 Oxacillin disk diffusion method: - Oxacillin disk $(1 \mu \mathrm{g})$ diffusion method was carried out on MuellerHinton Agar (MHA) supplemented with 2\% Nacl to detect MRSA according to CLSI guidelines. The plates were incubated at $35^{\circ} \mathrm{c}$ and results were recorded after 24hours of incubation. Isolates were considered resistant when the diameter of inhibition was $<10 \mathrm{~mm}$, intermediate resistance when the diameter was $10-12 \mathrm{~mm}$ and sensitive when the diameter was $>13 \mathrm{~mm} .{ }^{[7],[8]}$.

2.5 Detection using cefoxitin as a surrogate for oxacillin:- The Clinical and Laboratory Standards Institute (CLSI) guidelines (2006) has recommended cefoxitin disc diffusion method for the detection of MRSA. This

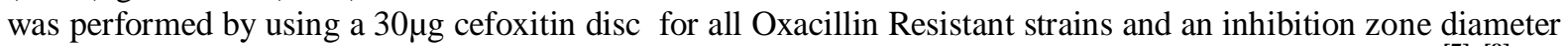
of $\leq 19 \mathrm{~mm}$ was considered as methicillin resistant and $\geq 20 \mathrm{~mm}$ was considered as methicillin sensitive ${ }^{[7],[8]}$.

2.6 Disk diffusion test with MH agar: The disk agar diffusion was performed by following the recommendations of the National Committee for Clinical Laboratory Standards (NCCLS). A sterile cotton swab was dipped in a suspension, which was adjusted to 0.5 McFarland turbidity. After the swab was streaked uniformly onto MH agar and Antibiotic sensitivity of the MRSA isolates against erythromycin $(15 \mu \mathrm{g})$, chloramphenicol $(30 \mu \mathrm{g})$, cotrimoxazole $(1.25 / 23.75 \mu \mathrm{g})$, ceftriaxone $(30 \mu \mathrm{g})$, gentamicin $(10 \mu \mathrm{g})$, Amoxicillin/clavulanic acid $(30 \mu \mathrm{g})$ and ciprofloxacin $(5 \mu \mathrm{g})$ was determined by modified Kirby-Bauer method and incubated at $35^{\circ} \mathrm{C}$ for $18-24 \mathrm{~h}$. Antibiotic sensitivity of VRSA strains were not tested against quinupristin/dalfopristin (Synercid) $(15 \mu \mathrm{g})$ because Isolates with inhibition zone diameter $\leq 7 \mathrm{~mm}$ around vancomycin disc was absent and not confirmed as Vancomycin Resistant Staphylococcus Aureus (VRSA)

All inocula on MHA were suspension of pure isolates in physiologic saline with density equivalent to a 0.5 McFarland turbidity standard. Staphylococcus aureus ATCC 25923 was used as control. Antibiotic sensitivity testing and result interpretation will be according to CLSI Standards.

\section{Results}

A total of 120 Health Care workers (HCWs), age range 20-50 years were screened for MRSA Carriage. Among them 77(64.1\%) were Males and 43(35.8\%) were Females. 40(33.3\%) Doctors, 65(54.1\%) Nursing staff and 15(12.5\%) Technicians are included in this study (Table 1). In all 120, 19 health Care workers were Positive for MRSA either in Nose, Palm and Web spaces or in three sites giving a carriage rate of $\mathbf{1 5 . 8 \%}$.And in remaining 101 health Care workers Coagulase negative Staphylococcus aureus (CONS) was isolated. The proportion of health Care workers with nasal carriage was higher $8(6.7 \%)$ than wed space $6(5 \%)$ and palm 5 (4.1\%) and no dual carriage and carriage in three sites was observed (Table 2). Among the 77 of males screened 5(6.4\%) were positive, compared to 43 females 14(32.5\%) of them were positive. The distribution of MRSA carriage across cadre in Doctors (2.5\%) Nursing staff (7.5\%) and Technicians (5.8\%) were colonized with MRSA (Table 3). Antibiogram was determined by Kirby Bauer disk diffusion method for all MRSA isolates. The MRSA isolates were sensitive to all antibiotics tested. The antibiotic sensitivity profiles in the three sites were: - (Table 4).

All the MRSA isolates of the three sites Nose, Palm and Web space were sensitive to all antibiotics tested and the isolate resistant to Vancomycin was not identified. 
Table: 1 Distribution of Participants

\begin{tabular}{||c|c||}
\hline \multicolumn{1}{|c|}{ PARAMETER } & DESCRIPTION \\
\hline AGE YEARS & FREQUENCY $(\%)$ \\
\hline $\mathbf{2 0 - 2 9}$ & $\mathbf{6 8}(\mathbf{5 6 . 6})$ \\
\hline $\mathbf{3 0 - 3 9}$ & $\mathbf{3 1}(\mathbf{2 5 . 8})$ \\
\hline $\mathbf{4 0 - 4 9}$ & $\mathbf{9}(\mathbf{7 . 5})$ \\
\hline $\mathbf{5 0 - 5 9}$ & $\mathbf{1 2}(\mathbf{1 0})$ \\
\hline$(2) \quad$ MEXDISTRIBUTION & $\mathbf{7 7}(\mathbf{6 4 . 1})$ \\
\hline Males & $\mathbf{4 3}(\mathbf{3 5 . 8})$ \\
\hline Females & $\mathbf{4 0}(\mathbf{3 3 . 3})$ \\
\hline$(3) \quad$ CATEGORYOF WORK & $\mathbf{6 5}(\mathbf{5 4 . 1})$ \\
\hline Doctors & $\mathbf{1 5}(\mathbf{1 2 . 5})$ \\
\hline Nurses & \\
\hline Technicians & \\
\hline
\end{tabular}

Table : 2 Site Distribution Of MRSA Isolates

\begin{tabular}{||l|c||}
\hline SITE DISTRIBUTION OF MRSA ISOLATES & FREQUENCY(\%)N=120 \\
\hline MRSA in Nose & $8(6.7)$ \\
\hline MRSA in Palm & $5(4.1)$ \\
\hline MRSA in Web space & $6(5.0)$ \\
\hline Either Nose, Palm and Web space & $19(15.8)$ \\
\hline
\end{tabular}

Table:3 Profession/Cadre Related Distribution Of MRSA Carriage

\begin{tabular}{||c|c|c|c|c||}
\hline & & & CADRE CARRIAGE RATE & \\
\hline CADRE & NO.SAMPLED & NO.POSITIVE & WITH IN CADRE & TOTAL(N=120) \\
\hline Doctors & $\mathbf{4 0}$ & $\mathbf{3}$ & $\mathbf{7 . 5}$ & $\mathbf{2 . 5}$ \\
\hline Nurses & $\mathbf{6 5}$ & $\mathbf{9}$ & $\mathbf{1 3 . 8}$ & $\mathbf{4 6 . 5}$ \\
\hline Technicians & 15 & 7 & & $\mathbf{5 . 8}$ \\
\hline TOTAL & 120 & 19 & & 15.8 \\
\hline
\end{tabular}

Table: 4 Antibiotic sensitivity profiles of MRSA isolates

\begin{tabular}{|c|c|c|c|c|}
\hline S.No. & Name of Antibiotic & $\begin{array}{l}\text { Nose } \mathrm{n}= \\
\mathbf{8 \%}(\mathbf{6 . 7})\end{array}$ & $\begin{array}{l}\text { Palm } \mathbf{n}=\mathbf{5 \%} \\
\mathbf{( 4 . 1 )}\end{array}$ & $\begin{array}{l}\text { Web space } \\
\mathrm{n}=\mathbf{6 \%}(\mathbf{5})\end{array}$ \\
\hline $\mathbf{1}$ & Gentamicin & $\mathbf{5 0 \%}$ & $\mathbf{6 0 \%}$ & $\mathbf{6 6 . 6 \%}$ \\
\hline $\mathbf{2}$ & Chloramphenicol & $\mathbf{6 2 . 5 \%}$ & $\mathbf{8 0 \%}$ & $\mathbf{5 0 \%}$ \\
\hline $\mathbf{3}$ & Co-trimoxazole & $\mathbf{6 2 . 5 \%}$ & $\mathbf{6 0 \%}$ & $\mathbf{8 3 . 3 \%}$ \\
\hline $\mathbf{4}$ & Erythromycin & $\mathbf{7 5 \%}$ & $\mathbf{6 0 \%}$ & $\mathbf{3 3 . 3 \%}$ \\
\hline $\mathbf{6}$ & Ciprofloxacin & $\mathbf{8 7 . 5 \%}$ & $\mathbf{8 0 \%}$ & $\mathbf{8 3 . 3 \%}$ \\
\hline $\mathbf{6}$ & Ceftriazone & $\mathbf{3 7 . 5 \%}$ & $\mathbf{4 0 \%}$ & $\mathbf{5 0 \%}$ \\
\hline $\mathbf{8}$ & Amoxyclavulanate & $\mathbf{1 0 0 \%}$ & $\mathbf{1 0 0 \%}$ & $\mathbf{1 0 0 \%}$ \\
\hline
\end{tabular}

\section{Discussion}

Colonization and infection by $S$. aureus are known to be significantly associated with infection among hospitalized patients. In the era of high prevalence of methicillin resistance among $S$. aureus, a relatively high percentage (11-19\%) of MRSA nasal carriage among hospital patients on admission will increase the likelihood of MRSA infection during the same episode of hospitalization ${ }^{[9]}$.

Methicillin resistance among Staphylococci is widespread in India. In a multicentre study from India in 1996, prevalence of MRSA ranged from 27\% in Bombay to $47 \%$ in Bangalore and $42.5 \%$ in Delhi. A study from Eastern India in 2001 revealed $66.6 \%$ of S. epidermidis and $60 \%$ of $S$. haemolyticus strains resistant to oxacillin. The most recent data from India gives a figure of $66 \%$ methicillin resistant CoNS among neonatal septicaemic patients; isolated from blood and skin of these patients ${ }^{[10]}$.

Studies on MRSA in intensive care units have also demonstrated that MRSA colonization predisposed to MRSA infection during the same hospitalization ${ }^{[11]}$. Upon discharge back to the community, a high Percentage $(29 \%)$ of previously infected patients were documented to develop infection during 18-month follow-up ${ }^{[12]}$. This greatly raises concerns about community infections caused by healthcare-associated strains 
of MRSA. Community-acquired MRSA (CA-MRSA) is potentially a new emerging pathogen with most strains susceptible to many antimicrobials except for $\beta$-lactam antibiotics.

In our study a total of 120 subjects age range between 20-50 years were screened for MRSA carriage. Among them 77(64.1\%) were males and 43(35.8\%) were females. From these subjects 40(33.3\%) Doctors, 65(54.1\%) Nursing staff and 15(12.5\%) were Technicians. Among 120 health Care workers screened, 19 were positive for MRSA either in nose, palm and web spaces or in three sites giving a carriage rate of $\mathbf{1 5 . 8 \%}$. And in remaining 101 health Care workers Coagulase negative Staphylococcus aureus (CONS) was isolated. The proportion of health Care workers with nasal carriage was higher $8(6.7 \%)$ than wed space $6(5 \%)$ and palm 5 $(4.1 \%)$ and no dual carriage and carriage in three sites was observed. Among the 77 of males screened 5(6.4\%) were positive, compared to 43 females 14(32.5\%) of them were positive. The distribution of MRSA carriage across cadre (presented table 3 ) in Doctors (2.5\%) Nursing staff (7.5\%) and Technicians (5.8\%) were colonized with MRSA.

Anand et.al ${ }^{[7]}$ from Pune in 2009 compared cefoxitin disc diffusion test with oxacillin agar screening and detection of $m e c A$ gene by PCR. Fifty strains of $S$. aureus +isolated from clinical samples were isolated by routine antibiotic susceptibility testing Out of the 50 isolates, 28 were found to be methicillin resistant by oxacillin disc diffusion test, 30 were resistant by oxacillin screen agar method, and 32 were resistant with cefoxitin disc diffusion. For these 32 isolates mecA gene was positive. In these study results of cefoxitin disc diffusion test is in concordance with the PCR for mecA gene. Thus, this test can be an alternative to PCR for detection of MRSA in resource constraint settings

Chacko et.al ${ }^{[13]}$ from Mangalore in 2008 studied for MRSA in Human immunodeficiency virus infected patients. They detected MRSA by agar screen and agar dilution methods. 46 of 60 (76.67\%) outpatients with HIV infection were colonized with S.aureus in the anterior nares. Significant numbers of S.aureus were in 31-40 year age group. Methicillin resistance was found in eight (17.39\%) isolates. Of the 46 S.aureus strains, 29 (63\%) were resistant to erythromycin, $69.5 \%$ to Co-trimoxazole and $41.3 \%$ to Ciprofloxacin. Co-trimoxazole use was found to be a risk factor for S.aureus carriage $(\mathrm{P}=0.0214)$ but not for methicillin resistance. From this study it is clear that prolonged hospital stay was a risk factor for methicillin resistance whereas stay for more than 25 days was found to be a highly significant risk factor. Dermatophytosis and herpes simplex virus infection were other risk factors for nasal carriage of S.aureus.

Mathanraj et.al ${ }^{[14]}$ from Pondicherry in 2008 screened 200 subjects (90 inpatients, 53 outpatients, and 57 HCWs) for carriage of Methicillin resistant Staphylococci aureus (MRSA) at different sites using oxacillin blood agar and Mannitol salt agar with oxacillin. Overall carriage rate was $8.5 \%$, with the highest rate in inpatients $(15.6 \%)$ while the lowest in HCWs (1.8\%) in these patients the commonest site of colonization of MRSA was the anterior nares.

Fadeyi et.al ${ }^{[2]}$ from Nigeria in 2011 screened 198 Health Care workers(HCWs) for isolation of MRSA in Anterior naries and Hand by inoculating the collected swabs on to Mannitol Salt Agar(MSA). Standard tests were performed to identify Staphylococcus.aureus and MRSA was identified by combined oxacillin and cefoxitin discs diffusion method, 104 had MRSA either in the nose, hand or both giving a carriage rate of $52.5 \%$. In this study Nasal carriage (38.9\%) was higher than hand (25.3\%) and Doctors $(22.7 \%)$ were the predominant carriers when compared with Nurses (16.7\%). MRSA isolates were resistant to commonly available antibiotics. Sensitivity to Vancomycin was identified by vancomycin discs diffusion and vancomycin agar screen plating and Only $1(1.3 \%)$ of the nasal isolates was vancomycin resistant. In this study MRSA carriage was particularly high among the Doctors $(65.2 \%)$ and Nurses $(64.7 \%)^{[2]}$.

In our present study, the $S$. aureus colonization rate (15.8\%) in health care workers was more when compared to (8.5\%) in Pondicherry, but less when compared to (28.1\%) in Taiwan ${ }^{[9]},(27.3 \%)$ in the American Indian population ${ }^{[15]}$ and $52.5 \%$, in the study done in Nigeria health care settings. In one study from Saudi Arabia, an overall nasal carriage of $38.0 \%$ was observed, and the carriage rate varied in different age groups ${ }^{[16]}$. In an earlier study in Nigeria ${ }^{[9]}$, subjects from health care-related facilities Nasal carriage (38.9\%) was higher than hand $(25.3 \%)$ and Doctors $(22.7 \%)$ were the predominant carriers when compared with Nurses $(16.7 \%)$. Our study also showed a higher MRSA carriage rate, in nasal carriage and was higher $8(6.7 \%)$ than wed space $6(5 \%)$ and palm $5(4.1 \%)$.

This indicates the necessity for further emphasis on hand hygiene for HCWs especially before going home, similar to recommendations currently given to patients and their families, to avoid transmission of MRSA to family members. These findings also point to the importance of eradicating MRSA colonization in HCWs and their family members, and their family contacts. Since MRSA infection is a potentially severe event, early diagnosis is essential. Prevention is thus important and relies on hygienic measures and strict protocols based on aseptic manipulation by HCWs. In nasal carriers of MRSA, eradication of bacteria by a topical antimicrobial ointment has been associated with a significant reduction of the incidence of bacteriaemias ${ }^{[17]}$.

In the present study, we found a higher proportion of MRSA carriage among the Nursing staff $(7.5 \%)$ when compared to Technicians (5.8\%) and least among Doctors $(2.5 \%)$. We have found only one study in which 
highest rate is found in inpatients $(15.6 \%)^{[14]}$. In contrast this study has found that the carriage rate was higher among the nursing staff. This may be due to geographic variation coupled with unhygienic practices in the individuals in the present study.

Locally applied or synthetic antibiotics are most commonly used to treat MRSA. Nasal ointments or sprays and oral antibiotics have variable efficacy and their use frequently results in antimicrobial resistance among S. aureus strains. Of the commonly used agents, Mupirocin (Pseudomonic acid) ointment has been shown to be $97 \%$ effective in reducing S. aureus nasal carriage ${ }^{[11]}$.

Indiscriminate use of antibiotics may lead to resistance to all the other groups of antibiotics. A very few MRSA resistant to Vancomycin and /or Teicoplanin have been found in the USA. Though our study found $100 \%$ sensitivity to Vancomycin, improper and indiscriminate use may in near future lead to resistance to these drugs all over the world. So use of appropriate antibiotic treatment in order to avoid the spread of multidrug resistant strains is recommended.

\section{Conclusion}

Health care workers do not appear to be a major source of MRSA in our hospital, although it would require screening of larger numbers before arriving at any definite conclusions. In corroboration with the above

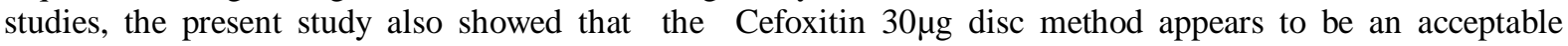
alternative for rapid identification of MRSA. However, further confirmatory study can be done by detection of $m e c A$ by PCR method, which is considered as the gold standard. There is need for the development, adoption and enforcement of appropriate control policies in hospitals with many points of critical care like our own where there are no existing or effective MRSA control programs.

\section{Acknowledgement}

This study was carried out as a short term project granted by the Indian Council of Medical Research (ICMR) to undergraduate students of Medicine (M.B.B.S). We are also grateful to Dr. Jampala Srinivas for Providing some important information included in this study.

\section{References}

[1] Kumar P, Shukla I, Varshney S Nasal screening of healthcare workers for nasal carriage of coagulase positive MRSA and prevalence of nasal colonization with staphylococcus aureus , Biology and medicine 27(1), 2011,62-64.

[2] A. Fadeyi, B.O.Bolaji, O.O.Oyedepo Methicillin resistant staphylococcus aureus, carriage among health care workers of critical care units in a Nigerian hospital , American Journal of Infectious Diseases 6(1), 2010, 18-23.

[3] Methicillin resistant Staphylococcus aureus. Wikipedia, the free encyclopedia, www.wikipedia.com.

[4] Nour de San, Olivier Denis Marie-Fabrice Gasarira, Ricardo De Mendonca, et al, Controlled Evaluation of the IDI-MRSA Assay for Detection of Colonization by Methicillin-Resistant Staphylococcus aureus in Diverse Musculocutaneous Specimens, Journal of Clinical Microbiology 45,2007,1098-1101.

[5] Hetty E.M. Blok, Annet Trolestra, Titia E.M.Kamp-Hopmans, etal.,- Role of health care workers in outbreaks of Methicillinresistant Staphylococcus aureus: A 10- year evaluation from a dutch university hospital;2003:24(9):679-685.

[6] Bruno Gonzalez-Zorn, Jose P. M. Senna, Laurence Fiette, Spencer Shorte, Aurelie Testard, Michel Chignard, Patrice Courvalin, and Catherine Grillot-Courvalin, Bacterial and Host Factors Implicated in Nasal Carriage of Methicillin-Resistant Staphylococcus aureus in Mice. Infection and Immunity, 73, 2005, 1847-1851.

[7] KB Anand, P Agrawal, S Kumar, K Kapila. Comparison of cefoxitin disc diffusion test, oxacillin screen agar, and PCR for mecA gene for detection of MRSA, Indian Journal of Medical Microbiology; 27(I), 2009, 27-29.

[8] Peacock J Sharon. Staphylococcus. In: Topley \& Wilson's Principles of Bacteriology, Virology and Immunology 10th edition, (2, Ed: Borriello SP, Murray PR, Guido Funke. 2005 771-810. Edward Arnold, USA.)

[9] Po-Liang Lu, Jer-Chia Tsai, Yi-Wen Chiu, Feng-Yee Chang, Ya-Wei Chen, Chin-Fu Hsiao and L. K. Siu, Methicillin-resistant Staphylococcus aureus carriage, infection and transmission in dialysis patients, healthcare workers and their family members, Nephrology Dialysis Transplant 23,2008, 1659-1665.

[10] Chaudhury A, Kumar AG. In vitro activity of antimicrobial agents against Oxacillin resistant Staphylococci with special reference to Staphylococcus haemolyticus. Indian Journal of Medical Microbiology 25(1),2007,50-52.

[11] TM Perl and JE Golub. New approaches to reduce Staphylococcus aureus nosocomial infection rates: treating S. aureus nasal carriage. The Annals of Pharmacotherapy: 32,1998,S7-16.

[12] Huang SS, Platt R. Risk of Methicillin-Resistant Staphylococcus aureus Infection after Previous Infection or Colonization .Clinical Infectious Diseases 36,2003,281-5.

[13] J Chacko, M Kuruvila, *GK Bhat- Factors Affecting -The Nasal Carriage of Methicillin Resistant Staphylococcus aureus In Human Immunodeficiency Virus-Infected Patients , Indian Journal of Medical Microbiology 27(2),2009, 146-8.

[14] S Mathanra, *S Sujatha, K Sivasanangeetha, SC Parija, Screening for Methicillin-Resistant Staphylococcus aureus Carriage among Patients and Health Care Workers Of a Tertiary Care Hospital In South India, Indian Journal of Medical Microbiology 27(1),2009,62-64.

[15] Leman R, varado-Ramy F, Pocock S et al. Nasal carriage of methicillin-resistant Staphylococcus aureus in an American Indian population. Infection Control and Hospital Epidemiology, 25,2004,121-125.

[16] Saxena AK, Panhotra BR. The prevalence of nasal carriage of Staphylococcus aureus and associated vascular access-related septicemia among patients on hemodialysis in Al-hasa region of Saudi Arabia. Saudi Journal of Kidney Disease Transplant 14,2003, 30-38.

[17] Tordoir J, Canaud B, Haage P et al. EBPG on vascular access. Nephrology Dialysis Transplant22 (Suppl 2) ii,2007,88-117. 